\title{
UTILIZAÇÃO PARALELA DE FITORREMEDIAÇÃO E BIORREMEDIAÇÃO PARA TRATAMEN- TO DE ÁREAS CONTAMINADAS
}

\author{
Dailleny Chagas de Oliveira Mariano \\ Graduada em Engenharia Civil pelo CEFET-RJ, RJ, Brasil \\ daillenymariano@gmail.com
}

Igor Barcelos Rodrigues

Graduando em Engenharia Civil pelo CEFET-RJ, RJ, Brasil

igorbarod@gmail.com

Marcelo de Jesus Rodrigues da Nóbrega

Pós Doutor em Engenharia pela UERJ, Rio de Janeiro, RJ, Brasil

Professor da Engenharia Civil do CEFET-RJ, RJ, Brasil

Professor da Universidade Santa Úrsula, Rio de Janeiro, RJ, Brasil

engmarcelocefet@terra.com.br

\section{RESUMO}

O presente artigo aborda duas ações mitigadoras, fitorremediação e biorremediação, para tratamento de áreas contaminadas. Faz análise dos métodos e suas aplicações e como estes podem ser utilizados de maneira paralela para auxiliar o tratamento das áreas contaminadas. Intensifica a redução do tempo utilizado para o tratamento e aumenta a eficácia das ações. Explicita a situação atual do tratamento de áreas contaminadas e as normas as quais a regem. Traça um panorama para melhor desempenho do tratamento de áreas contaminadas por meio dos processos biológicos.

Palavras-chave: Áreas contaminadas. Processos Biológicos. Biorremediação. Fitorreme- 
diação.

\title{
PARALLEL USE OF PHYTEMEDICATION AND BIORREMEDIATION FOR THE TREATMENT OF CONTAMINATED AREAS
}

\begin{abstract}
This article discusses two mitigating actions, phytoremediation and bioremediation, for the treatment of contaminated areas. It analyzes the methods and their applications and how they can be used in parallel to assist the treatment of contaminated areas. It intensifies the reduction of the time used for the treatment and increases the effectiveness of the actions, since the parallel methods can be used to obtain more favorable results. Explains the current status of the treatment of contaminated areas and the rules that govern it. It outlines a better performance of the treatment of contaminated areas through biological processes.
\end{abstract}

Keywords: Contaminated areas. Biological Processes. Bioremediation. Phytoremediation.

\section{INTRODUÇÃO}

A preocupação com o meio ambiente e sua preservação tem sido um tema de relevância considerada nos últimos anos. De acordo com o Instituto Estadual do Ambiente - INEA (2018) o Estado do Rio de Janeiro se desenvolveu industrialmente de forma rápida e sem a adequada cautela com o meio ambiente. Desta forma sem controle e planejamento as atividades poluidoras ocasionaram contaminação em inúmeras áreas tanto no solo quanto em águas subterrâneas.

O Ministério do Meio Ambiente - MMA (Brasil, 2018) define como área contaminada: 
[...] área, terreno, local, instalação, edificação ou benfeitoria que contenha quantidades ou concentrações de quaisquer substâncias ou resíduos em condições que causem ou possam causar danos à saúde humana, ao meio ambiente ou a outro bem a proteger, que nela tenham sido depositados, acumulados, armazenados, enterrados ou infiltrados de forma planejada, acidental ou até mesmo natural. (BRASIL, 2018).

O MMA (Brasil, 2018) declara que os agentes poluidores podem estar em solos, sedimentos, rochas, águas subterrâneas, entre outras e desta maneira podem se concentrar em partes das edificações como pisos, paredes e estruturas. Sendo assim, podem ser conduzidos a outros lugares próximos a ponto de contaminá-los.

Visando o controle, planejamento e maior fiscalização dessas atividades potencialmente poluidoras o Conselho Nacional do Meio Ambiente - CONAMA elaborou a Resolução o 420 de 28 de dezembro de 2009 (Brasil, 2009) em prol de evitar riscos ao meio ambiente e a saúde pública. Esta resolução dispõe sobre:

[...] critérios e valores orientadores de qualidade do solo quanto à presença de substâncias químicas e estabelece diretrizes para o gerenciamento ambiental de áreas contaminadas por essas substâncias em decorrência de atividades antrópicas (BRASIL, 2018).

Esta tem como característica garantir que seja feita a proteção do solo, com manutenção visando recuperar a qualidade deste e deixá-lo apropriado para uso e para tal definem-se os processos aos quais devem ser executados. Esta resolução é de domínio federal e trata a respeito do gerenciamento de áreas contaminadas impondo aos estados a responsabilidade de cadastrar e divulgar publicamente áreas classificadas como contaminadas.

O CONAMA define no Art $6^{\circ}$ da Resolução o 420 de 28 de dezembro de 2009(Brasil, 2009) que dentre as etapas envolvidas para a sondagem de uma área estão as avaliações de risco e preliminar que tem como objetivo identificar e analisar se área está ou não contaminada, quais os riscos à saúde humana e classificá-la de acordo com o contaminante.

Em prol de um controle e fiscalização a respeito deste assunto a Associação Brasi- 
leira de Normas Técnicas - ABNT elaborou a norma NBR no 15515 (ABNT, 2011) que tem como intuito o passivo ambiental em solo e água e está dividida em três partes por meio que a parte 1 relata a avaliação preliminar, a parte 2 relata a investigação confirmatória e a parte 3 relata a respeito da investigação detalhada.

A presente norma contém medidas mitigadoras referentes a contaminação de áreas tanto no solo quanto na água e o presente trabalho tem como intuito abordar duas ações mitigadoras a respeito de áreas contaminadas, a fitorremediação e a biorremediação, com o propósito de definir quando se faz melhor o uso destas ou quando estas podem ser associadas para um melhor desempenho.

\section{DESENVOLVIMENTO}

O Instituto de Pesquisas Tecnológicas do Estado de São Paulo - IPT possui um Guia de elaboração de planos de intervenção para o gerenciamento de áreas contaminadas (Moraes, Teixeira e Maximiano, 2014) que contém os detalhamentos das etapas a serem seguidas para o tratamento de áreas e a descrição de como devem ser feitas. Dentre as medidas de remediação os processos estão separados em químicos, biológicos e térmicos.

Os processos biológicos podem ser tratados por meio da biorremediação ou da fitorremediação que serão abordados a seguir.

\subsection{Biorremediação}

Biorremediação é o processo de remover ou reduzir a quantidade de contaminantes em um ambiente com uso de organismos vivos, normalmente plantas, microrganismos ou suas enzimas. É uma técnica bastante eficiente para o tratamento de moléculas recalcitrantes - de difícil degradação, naturais ou xenobióticas - estranhas ao ambiente natural, de forma ecologicamente mais adequada (GAYLARDE 2005; ANDRA- 
DE, AUGUSTO, JARDIM 2012).

Como os microrganismos desempenham a tarefa de reciclar a maior parte das moléculas da biosfera, o sistema microbiano permite a biodegradação de substâncias perigosas, sem que haja a necessidade de transferir o contaminante de um meio para o outro, o que influi para baixo custo de execução, se comparado com outras técnicas de remediação. Além disso a biorremediação possui maior eficiência em meios homogêneos e de textura arenosa e se os compostos forem facilmente degradáveis a tecnologia pode ser considerada como destrutiva dos contaminantes (GAYLARDE 2005; CETESB 2007).

Porém, de acordo com a CETESB(2007) para aplicação deste processo é necessária a avaliação de alguns fatores, como:

- a suscetibilidade do contaminante à degradação;

- presença de populações microbiológicas apropriadas e em quantidades suficientes para promover uma taxa de degradação adequada;

- condições do pH e temperatura para o crescimento dos microrganismos;

- biodisponibilidade do contaminante;

- geração de subprodutos que sejam menos tóxicos que os produtos primários;

- capacidade do meio de sustentar atividade biológica.

Portanto torna-se necessária uma análise criteriosa e individual da área a ser remediada para escolha da melhor técnica a ser utilizada.

O quadro 1 descreve as vantagens e desvantagens entre as possíveis técnicas de utilização do processo de biorremediação.

Assim é possível notar que a aplicação da biorremediação é uma variável que dispõe das características do solo tanto quanto da localidade e necessita de uma avaliação quanto as desvantagens referentes a cada técnica aplicada. 


\begin{tabular}{|c|c|c|}
\hline Técnica & Vantagens & Desvantagens \\
\hline In situ & $\begin{array}{l}\text { Evita custos e distúrbios ambientais associa- } \\
\text { dos ao movimento do material contaminado } \\
\text { para o tratamento. }\end{array}$ & $\begin{array}{l}\text { Manipulação limitada do meio } \\
\text { contaminado. }\end{array}$ \\
\hline Passiva & $\begin{array}{l}\text { Apresenta um baixo custo e ajuda a minimi- } \\
\text { zar os riscos para a saúde humana e meio } \\
\text { ambiente, por ser um processo natural. }\end{array}$ & $\begin{array}{l}\text { Pode ser lento, necessitando } \\
\text { de outras técnicas. }\end{array}$ \\
\hline Bioestimulação & $\begin{array}{l}\text { Grande disponibilidade dos produtos a se- } \\
\text { rem utilizados e um baixo custo. }\end{array}$ & $\begin{array}{l}\text { Pode necessitar de repetidas } \\
\text { aplicações, devido à solubili- } \\
\text { dade. }\end{array}$ \\
\hline Bioventilação & $\begin{array}{l}\text { Minimização da extração de vapores, equi- } \\
\text { pamentos de fácil instalação e aquisição, } \\
\text { atuação em locais de difícil acesso e peque- } \\
\text { no impacto na área contaminada. }\end{array}$ & $\begin{array}{l}\text { Baixa umidade, temperaturas } \\
\text { amenas e solos com pouca } \\
\text { permeabilidade, podem limi- } \\
\text { tar a eficiência da técnica. }\end{array}$ \\
\hline Bioaumentação & $\begin{array}{l}\text { Intensificar o processo de biodegradação do } \\
\text { contaminante. }\end{array}$ & Riscos ambientais \\
\hline Ex situ & $\begin{array}{l}\text { Resultado rápido, maior versatilidade para o } \\
\text { tratamento de vários contaminantes. }\end{array}$ & Alto custo. \\
\hline Landfarming & $\begin{array}{l}\text { Tempo pequeno de tratamento, custo com- } \\
\text { petitivo, eficiente para constituintes orgâni- } \\
\text { cos com baixa biodegradabilidade. }\end{array}$ & $\begin{array}{l}\text { Pouco eficiente para consti- } \\
\text { tuintes pesados, necessita de } \\
\text { grandes extensões de terra, } \\
\text { geração de poeira e vapores. }\end{array}$ \\
\hline
\end{tabular}

Fonte: (Adaptado de Jacques et al. (2006), Mariano (2006), Corseuil e Marins (1998), CETESB - online, ABBAS (2003) e TORCHETTO (2008) apud Rocha e Alves (2013))

\subsection{Fitorremediação}

A fitorremediação é um tipo de tratamento do solo e da água que utiliza plantas para degradar, extrair, conter ou imobilizar contaminantes. Muitas das técnicas da fitorremediação são baseadas em conhecimentos já existentes na agricultura, silvicultura e horticultura, com sua aplicação direcionada para o tratamento de problema ambientais (EPA, 2000).

Essa técnica é considerada uma alternativa limpa, pois gera mínima degradação e desestabilização da área, baixo impacto ambiental e garante uma estética favorável ao local em que é aplicada. Além de possuir um baixo custo de investimento e operação, sendo menos dispendiosa do que outras técnicas de extração de contaminantes. (Chaves, Mesquita, Araujo \& França, 2010)

A efetividade da fitorremediação depende principalmente do grau de contami- 
nação do metal no solo, da capacidade das plantas em acumularem o metal na parte aérea e da disponibilidade do metal no solo. Porém esses metais podem ser solubilizados pela adição de agentes complexantes, permitindo aumento da captação das plantas. (ERNEST, 1996 ; Doumett, Lamperi, Checchini, Azzarello, Mugnai, Mancuso, et al. 2008) Outros agentes limitantes que interferem no tempo necessário para a descontaminação são o clima, o tipo de solo, a estação do ano, a concentração e profundidade do contaminante e a interferência do contaminante no crescimento da planta. (Vasconcellos, 2012 apud Cunningham \& Ow, 1996; Pilon-Smits, 2005).

A fitorremediação não é aconselhada para áreas que necessitem de um tratamento rápido por oferecerem riscos aos seres vivos, pois esse processo pode levar semanas, meses e até anos para que se atinja o efeito esperado. Apesar de que existam métodos para melhorar seus resultados, como o melhoramento genético das plantas, os resultados ainda não são imediatos (EPA, 2000).

De acordo com Silva, Santos e Gomes (2014), a fitorremediação pode ser dividida em três técnicas com finalidades mais específicas:

a) Fitoextração: Uso de plantas para remoção de metais dos solos mediante absorção pelas raízes, transporte e concentração na biomassa da parte aérea;

b) Fitoestabilização: Uso de plantas para minimizar a mobilidade de metais em solos contaminados mediante a acumulação nas raízes ou precipitação na rizosfera;

c) Fitovolatilização: Baseado na capacidade das plantas volatilizarem metais do solo, aplicável, por exemplo, a Se e Hg.

Na figura 1 é possível visualizar o funcionamento de alguns desses processos, a fitoestimulção será explicada mais a frente. 
Figura 1 - Principais mecanismos e vias de transporte dos contaminantes envolvidos na fitorremediação

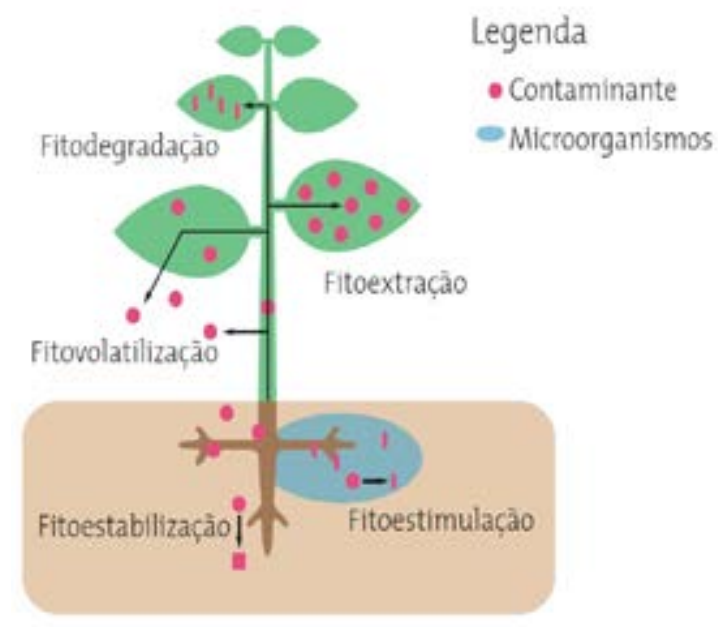

Fonte: (Guia de elaboração de planos de intervenção para o gerenciamento de áreas contaminadas Adaptado de PILON-SMITS, 2005)

Por meio destas técnicas procura-se obter o melhor resultado referente às necessidades do local em que o solo se encontra.

\section{ANÁLISE PARALELA ENTRE OS MÉTODOS}

Microrganismos e plantas podem ser aplicados individualmente para tratamento do solo, porém se combinados permitem a obtenção de maiores resultados em menor tempo. Determinados tipos de contaminantes podem ser mais difíceis de serem solubilizados e absorvidos pelas plantas por estarem muito integrados com a matéria orgânica do solo, ou os microrganismos podem ter dificuldades de degradar ou chegar ao local do contaminante. Visto essas dificuldades, algumas técnicas foram desenvolvidas a partir de fenômenos naturais observados da interação de plantas e microrganismos.

Um exemplo é a Rizodegradação, ou biodegradação de planta assistida, que é o aumento da atividade microbiana na quebra de contaminantes pela presença de plantas no local. A presença da zona radicular da planta aumenta a biodegradação na rizosfera 
(VASCONCELLOS, 2012).

A Rizodegradação é um processo de fitoestimulação que, segundo Vasconcellos (2012), consiste na liberação de aminoácidos e polissacarídeos pelas raízes das plantas, o que é considerado um estímulo para a atividade microbiana, além de que essas substâncias tem seu próprio potencial de degradar certos componentes do solo. Logo, é um processo muito útil para degradação de compostos orgânicos hidrofóbicos que não podem ser absorvidos pela planta, mas podem ser degradados por microrganismos.

Como observado no exemplo acima, a interação das plantas e microrganismos tende a gerar um benefício a ambos os lados, no que diz respeito a dissolução e absorção de contaminantes. O crescimento radicular da planta ajuda na abertura do solo para melhor infiltração de água e difusão de oxigênio, o que ajuda na atividade microbiana, enquanto a presença de micorrizas, associação entre fungos e a raiz, incrementa o crescimento da planta e favorece a dissipação de hidrocarbonetos de petróleo (GÜNTHER, DORNBERGER e FRITSCHE, 1996; KAIMI et al., 2006).

Segundo RUFYIKIRI, THIRY e DECLERCK (2003), foi realizado um experimento comparando a absorção de uranio do solo pelas raízes de uma planta, com e sem a presença de micorrizas. Foram utilizados o fungo "Glomus intraradices" e a planta "Daucus carota L.", dentro de ambientes controlados similares e com concentrações iguais de urânio, ao final do experimento observou-se que a absorção com micorrizas foi de 17,5\% da quantidade inicial, enquanto sem micorrizas foi de apenas $6,1 \%$.

A partir deste ponto de vista é possível verificar que a utilização de ambas às técnicas em paralelo pode intensificar o tratamento de uma determinada área contaminada desde que esta seja classificada e analisada dentro dos parâmetros para utilização dos dois métodos. Esta etapa é de suma importância, pois não serão todos os casos em que se poderá fazer a utilização de ambos os métodos. Uma vez que há outros referenciais em vista como o custo benefício. 
Quadro 2 - Vantagens e desvantagens da combinação das duas técnicas

\begin{tabular}{|c|c|}
\hline Vantagens & Desvantagens \\
\hline Maior taxa de absorção do contaminante & $\begin{array}{c}\text { Depende muito das caraterísticas do solo e } \\
\text { do clima }\end{array}$ \\
\hline Relações já existentes na natureza & Necessita maior estudo \\
\hline $\begin{array}{c}\text { Resultados mais rápidos se comparada ao } \\
\text { uso individual das duas técnicas }\end{array}$ & Necessita de superfície livre \\
\hline Grande variedade de plantas e fungos & Custo um pouco maior do que da técnica \\
individual
\end{tabular}

Fonte: (Autor)

\section{CONSIDERAÇÕES FINAIS}

As plantas e microrganismos já exercem naturalmente a tarefa de quebra e absorção dos materiais contaminantes, portanto o uso dessas técnicas é menos agressivo e gera um mínimo impacto ambiental. Porém, são técnicas lentas que propiciam contenção e tratamento do solo a longo prazo, essa característica pode ser contornada pela combinação de ambas as técnicas, que, se bem planejada pode gerar uma relação mutuamente benéfica entre os microrganismos e as plantas, aumentando a taxa de degradação e absorção do contaminante, o que diminui o tempo necessário para o tratamento.

Levando em consideração o clima, o pH do solo e o tipo de material contaminante, a escolha de uma planta e um microrganismo que sobrevivam as condições locais e que sejam mutuamente benéficas pode ser um desafio. Portanto essa técnica ainda carece de estudos mais aprofundados quanto as possíveis combinações.

\section{REFERÊNCIAS}

ANDRADE, Juliano de Almeida; AUGUSTO, Fabio; JARDIM, Isabel Cristina Sales Fontes. 
Biorremediação de solos contaminados por petróleo e seus derivados. Eclet. Quím., São Paulo, v. 35, n. 3, p. 17-43, Sept. 2010. Available from <http://www.scielo.br/scielo. php?script=sci_arttext \&pid=S0100-46702010000300002\&lng=en\&nrm=iso $>$. access on 06 Nov. 2018. http://dx.doi.org/10.1590/S0100-46702010000300002.

ASSOCIAÇÃO BRASILEIRA DE NORMAS TÉCNICAS. NBR 15515-1: passivo ambiental em solo e água subterrânea, Parte 1: Avaliação Preliminar. Disponível em

<http://licenciadorambiental.com.br/wp-content/uploads/2015/01/NBR-15.515-1-Passivo-Ambie

ntal-Avalia\%C3\%A7\%C3\%A3o-Preliminar.pdf> Acesso em 06 nov. 2018.

BRASIL. Resolução no 420, de 28 de dezembro de 2009. Dispõe sobre critérios e valores orientadores de qualidade do solo quanto à presença de substâncias químicas e estabelece diretrizes para o gerenciamento ambiental de áreas contaminadas por essas substâncias em decorrência de atividades antrópicas. Disponível em: < http://www2.mma. gov.br/port/conama/legiabre.cfm?codlegi=620>. Acesso em: 30 out. 2018.

CETESB -Companhia Ambiental do Estado de São Paulo. São Paulo, 1999. Disponível em: <http://www.cetesb.sp.gov.br/solo/areas_contaminadas/capitulo_x.pdf>, visualizado $12 / 11 / 2013$.

CHAVES, L. H. G., Mesquita, E. F., Araujo, D. L., \& França, C. P. (2010). Acúmulo e distribuição de cobre e zinco em mamoneira cultivar BRS Paraguaçu e crescimento da planta. Engenharia-Ambiental-Espirito Santo do Pinhal, 7(3), 263-277.

CUNNINGHAM, S. D., \& Ow, D. W. (1996). Promises and prospects of Phytoremediation. Plant Physiology, 110, 715-719. PMid:12226213.

DOUMETT, S., Lamperi, L., Checchini, L., Azzarello, E., Mugnai, S., Mancuso, S. et al. (2008). Heavy metal distribution between contaminated soil and Paulownia tomentosa, in a pilot-scale assisted phytoremediation study: Influence of different complexing agents. Chemosphere, 72, 1481- 1490. doi:10.1016/j.chemosphere.2008.04.083. 
EPA. (2000). Introduction of phytoremediation. EPA/600/R-99/107. Recuperado em 5 Maio 2012, de http://ne-pis.epa.gov.

ERNEST, W. H. O. Bioavailability of heavy metals and decontamination of soils by plants. Applied Geochemistry, v. 11, p. 163-167, 1996.

GAYLARD, C. C.; BELLINASO, M. L.; MANFIO, G. P. Aspectos biológicos e técnicas da biorremediação de xenobióticos. Biotecnologia, Ciência e Desenvolvimento, Brasília, v. 8, n. 34, jan./jun. 2005. Disponível em: <http://www1.esb.ucp.pt/twt/olimpiadasbio07/MyFiles/ MyAutoSiteFiles/FontesInformacao253906202/samorais/Biorremediacao.pdf>. Acesso em: 06 nov. 2018.

GÜNTHER, T.; DORNBERGER, U.; FRITSCHE, W. Effects of ryegrass on biodegradation of hydrocarbons in soil. Chemosphere, Oxford, v.33, n.2, p.203-215, 1996.

INEA, Instituto Estadual do Ambiente. Avaliação de Áreas Contaminadas. Disponível em < http://www.inea.rj.gov.br/Portal/Agendas/LicenciamentoAmbiental/Licenciamento-saiba-mais/GestaodeRiscoAmbientalTec/AvaliacaodeAreasContaminadas/index. htm\&lang=> Acesso em 30 out. 2018.

KAIMI, E. et al. Ryegrass enhancement of biodegradation in diesel-contaminated soil. Environmental and Experimental Botany, Oxford, v.55, n.1-2, p.110-119, 2006.

MINISTÉRIO DO MEIO AMBIENTE. Secretaria de Articulação Institucional e Cidadania Ambiental. Áreas Contaminadas. Disponível em < http://www.mma.gov.br/cidades-sustentaveis/residuos-perigosos/areas-contaminadas.html> Acesso em 30 out. 2018

MORAES, Sandra Lúcia; TEIXEIRA, Cláudia Echevenguá; MAXIMIANO, Alexandre Magno de Sousa. Guia de elaboração de planos de intervenção para o gerenciamento de áreas contaminadas. IPT - Instituto de Pesquisas Tecnológicas do Estado de São Paulo : BNDES, 2014. 1. ed. rev.. São Paulo. Disponível em <https://www.google.com/url?sa=t\&rct=j\& $q=\&$ esrc=s\&source $=$ web \&cd=10\&ved=2ahUKEwiLzqLVp9HeAhWGhpAKHc77CVYQFjAJ 
egQICBAC\&url=https\%3A\%2F\%2Fwww.ipt.br\%2Fdownload.php\%3Ffilename\%3D1317-Guia_Gerenciamento_de_Areas_Contaminadas 1a_edicao_revisada.pdf\&usg=A OvVaw252YUDmmVGCkeDNkt99F7J> Acesso em 13 nov. 2018

PILON-SMITS, E. (2005). Phytoremediation. Annual Review of Plant Biology, 56, 15-39. doi:10.1146/annurev.arplant.56.032604.144214.

ROCHA, C. A.; ALVES, F. Avaliação dos principais micro-organismos e técnicas utilizados na biorremediação de solos contaminados por petróleo e seus derivados, Centro Universitário Metodista Izabela Hendrix, 2013. Disponível em: <https://www.metodista.br/ revistas/revistas-izabela/index.php/aic/article/view/511>. Acesso em: 06 nov. 2018.

RUFYIKIRI, G.; THIRY, Y.; DECLERCK, S. Contribution of hyphae and roots to uranium uptake and translocation by arbuscular mycorrhizal carrot roots under root-organ culture conditions. New Phytologist, Oxon, v.158, n.2, p.391-399, 2003.

TAVARES, Silvio R. De L.. Capítulo 2: Técnicas de Remediação. Disponível em <https://ainfo.cnptia.embrapa.br/digital/bitstream/item/100359/1/Cap-2Livro-CA-Silvio-Tavares. pdf> Acesso em 06 nov. 2018.

VASCONCELLOS, M. C., Pagliuso D., Sotomaior, V. S. (2012) Fitorremediação: Uma proposta de descontaminação do solo. Estudos de biologia, v. 34, n. 83, p. 261-267, 2012. doi:10.7213/estud.biol.7338 